Original Research Article

\title{
Evaluating effectiveness of patient counseling, teach back versus standard method
}

\author{
Mary R. Mathew*, Linu Mohan, Mammen Paul, Mahira Maideen, \\ Liya Jose, Minnu Ommanakuttan
}

Department of Pharmacy Practice, Al Shifa College of Pharmacy, Malappuram, Kerala, India

Received: 01 November 2017

Accepted: 24 November 2017

*Correspondence to:

Dr. Mary R. Mathew,

Email: mary.rmathew@

yahoo.com

Copyright: (C) the author(s), publisher and licensee Medip Academy. This is an openaccess article distributed under the terms of the Creative Commons Attribution NonCommercial License, which permits unrestricted noncommercial use, distribution, and reproduction in any medium, provided the original work is properly cited.

\begin{abstract}
Background: The aim of the study was to assess memory retention of new prescription education by comparing Teach back method and standard counseling method. And also to evaluate association of age, sex, drug use in past and education in memory retention.

Methods: A prospective experimental study was carried out for a period of six months. Eligible subjects visiting pulmonary medicine outpatient department were screened and grouped into standard groups and teach back group. Patients in the standard group are taught eight counseling points about the drugs by one way dialogue method and asked at the end if there are any questions. while patients in the teach back method builds on the standard method by asking three open ended questions to recall what was taught and correcting any misunderstandings by two way dialogue method.

Results: The demographic information (age, sex, education, current prescription use) are collected from both groups. The post counseling score is assessed by evaluator using a scoring sheet. Scores were analyzed using Mann Whitney U test Teach back group shows statistically significant ( $\mathrm{p}$ value $=0.0001$ ) increase in score compared to standard group. The mean value of teach back scoring is 6.28 while that of standard is 4.44 .

Conclusions: All the demographic parameters (Age, sex, drug use in past three months and education) do not show any significant association with scoring and memory retention ( $\mathrm{p}$ value $>0.05$ for chi square test). The group that received teach-back method of counseling showed a significant improvement in patient knowledge and memory retention.
\end{abstract}

Keywords: Health literacy, Patient counseling, Pulmonary medicine, Patient education, Teach-back

\section{INTRODUCTION}

Health literacy is defined by the Institute of Medicine and the National Library of Medicine as "the degree to which individuals have the capacity to obtain, process, and understand basic health information and services needed to make appropriate health decisions". ${ }^{1,2}$ Patients are being treated with an ever-increasing array of medications and complex treatment regimens, which points towards the importance of health literature in today's world. Skills possessed by patients are not only the determinants of health literacy, but there are multitude of factors associated like complexity of healthcare system, culture, education, interaction between healthcare professionals and patients, complicated medication regimens, etc. ${ }^{3,4}$ Many studies over the years have found that adults have difficulty in understanding the instructions to take their medications. ${ }^{5-9}$ Evidences suggest that almost $46 \%-63 \%$ of the information provided to the patient is not retained by the patient. In another study, patients couldn't recollect the counseling information on discharge medication provided to them, just after they were discharged. These evidences proves that during most of the medical encounters the patient leaves the healthcare professional with poor 
understanding of their disease and medications. This can reduce patient compliance to treatment, which can lead to adverse health outcomes and decreased satisfaction. An estimated $\$ 73$ billion is lost annually due to misunderstood medical information.

Improving interpersonal communication has an important role to play when it comes to patient understanding. For improving the interpersonal communication, follow the six steps rule. The first step being slowing down-reduce the speed of communication, which can improve clinicianpatient interaction. Secondly, use plain and simple language to make them understand the concepts better. Thirdly, use visual aids to improve the understanding and patients recalling capacity. In the fourth step, always remember to limit the information provided and repeat it, for better recollection. In the next step, use teach-back technique - ask your patients to recall and repeat back your instructions. Finally, make the patients comfortable and encourage them to ask questions.

Teach back is a communication technique in which the patients are asked to recall back the information provided to them about their diagnosis and medication. Teach back approach is considered as one of the 34 proven safe practices by National Quality Forum and it is recommended by experts to adopt this technique as universal precaution due to difficulty in identifying patients having low health literacy. Poor health status can make even the highly skilled individuals vulnerable to misunderstanding of information provided to them. The use teach back techniques enable the patient to demonstrate the level of comprehension of medical counseling provided to them and can help them better understand their regimen and disease warning signs better. ${ }^{10,11}$ This becomes vital in patients with chronic health conditions as their understanding and recall on their condition and treatment is important for their health. The improved understanding can in turn improve the compliance with the therapy, which can reduce the hospital visits made by the patients. ${ }^{11}$ Teach back sessions can be made more effective by scheduling the session in advance, accessing the patient's baseline knowledge, involving family members in sessions and making the process a hands-on approach. The process of teach back should involve explaining the diagnosis and medication information to the patient and then assessing the patient's understanding with the information he recalls back. Based on this assessment the patient should be taught again for the information he couldn't understand and again access the patient through teach back.

Studies related to medical education and teach back technique are done mainly in developed countries like United States of America, Canada, Australia etc. where their vernacular is English. Medical terminologies and medical instructions are mainly written in English which is not understandable to common people especially in developing countries like India. So, the importance of medical education becomes evident when it comes to low health literacy regions like India. Not only the dissemination of knowledge is important, but also its retention by patients is equally important. As with the evidences seen from developed countries, teach back can be an inexpensive approach to improve the memory retention of the patient about their diagnosis and therapy. This common notion is put to test in our study; where we intend to evaluate the memory retention of a new prescription education by comparing teach back method and standard counseling method with additional objective of evaluating association of age, sex, drug use in past and education in memory retention.

\section{METHODS}

A prospective experimental study was carried out for a period of six months in the pulmonary medicine department of a 500 bedded multispecialty tertiary care referral hospital situated in Perinthalmanna, Malappuram district of Kerala. All Patients who were new to the pulmonary medicine outpatient units and above 18 years of age were included into the study irrespective of their gender. Patients with memory impairment, language or communication problem and healthcare professionals were excluded from the study. A total of 150 patients were enrolled into the study as per the protocol approved by the IEC of the institution. Data collection form was prepared based on the data required for evaluation which includes patient demographic details, drug use in three months, and education details. The patient data was collected from the outpatient treatment chart and personal interview with patient. A post counseling scoring sheet containing eight questions were developed after literature review and was validated by two experts (pulmonologist and clinical pharmacist).

\section{Sample size}

Sample size calculation was done by using formula:

$$
\mathrm{N}=\mathrm{Z} 21-\alpha / 2 \mathrm{p}(1-\mathrm{p}) / \mathrm{d} 2
$$

Z1- $\alpha / 2=$ value of the normal deviate at considered level of confidence (1.96)

$\mathrm{P}=$ expected prevalence of study group $(50 \%)$

$\mathrm{d}=$ expected absolute allowable error in P (10\%)

The minimum sample size was found to be 96 .

\section{Study procedure}

Eligible subjects visiting pulmonary medicine outpatient department were screened and randomized into standard groups and teach-back group. The new patients after obtaining the prescription from the physician would return with the medicines from the pharmacy to the pulmonary medicine OPD. The informed consent was obtained from patients who were willing to participate in the study. Then 
the patient is given prescription education by the students. Patients in the standard group are taught eight counseling points about the drugs by one way dialogue method (name, indication, dose, frequency, relation to food, avoidance of substance and two side effects) and asked at the end if there are any questions. while patients in the teach-back groups were counselled using a method that builds on the standard method by asking three open ended questions to recall what was taught and correcting any misunderstandings by two way dialogue method. The demographic information (age, sex, education, current prescription use) are collected from both groups. The post counseling score is assessed by a blinded evaluator using a scoring sheet with counseling point rephrased scoring 1 point each with a maximum score of 8 points.

\section{Data analysis}

Data collected during the study period was analyzed for association of age, sex, number of drugs used in three months and education with memory retention and for the effectiveness of teach back method over standard method. The association between age, gender, drug use in past three months and education with memory retention was determined using Pearson's chi square test. The overall scoring of teach back group and standard group was compared using Mann Whitney U test. Data analysis was performed using SPSS software version 20.

\section{RESULTS}

A total of 150 patients were enrolled into the six months study out of which 75 patients were enrolled into teachback group and the other 75 patients to the standard group. In the teach back group both male and female obtained the highest score of 8 but in the standard group males obtained the highest score of 8 but females highest score was 7 . In the teach-back group, $23.8 \%$ males scored 8 while $36.3 \%$ females 8 . In the standard group, $38.2 \%$ of the female population was having a score of 5 while each $19.5 \%$ of males were having a score of 3 and 4 (Figure 1).

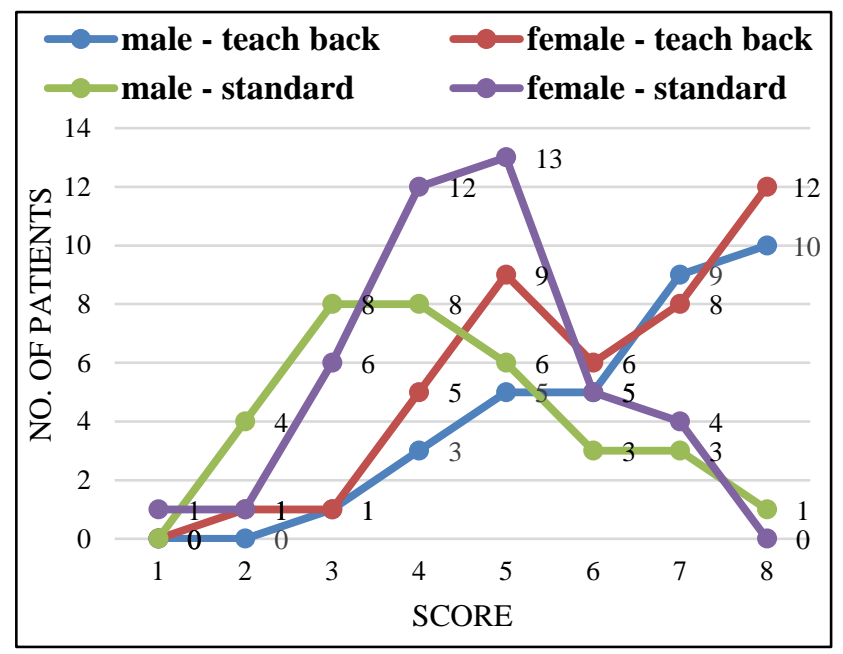

Figure 1: Gender v/s score: teach back and standard.
In the age group 18-55 of teach back, 8 is the highest total score $(24 \%)$ and in the 56- 89 age group, 5 is the highest total score $(10.6 \%)$. Eighteen patients scored 8 in the age group 18-55 and four patients in the age group 56-89 scored 8 . In the age group 18-55 of the standard group, 5 is the highest total score $(21.33 \%)$ and in the 56-89 age group the highest total score is $3(12 \%)$. One person scored 8 in the age group 18-55 and none in the age group 56-89 scored 8.4 (Figure 2).

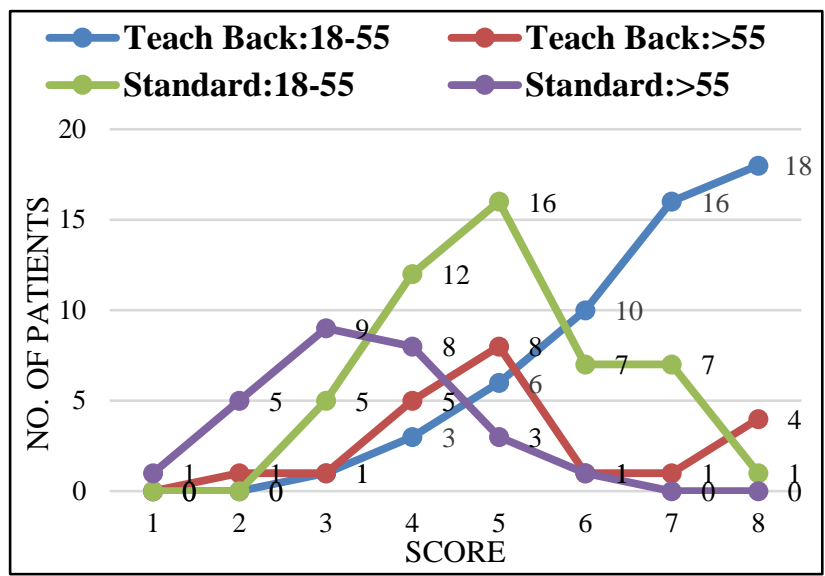

Figure 2: Age (in years) v/s score: teach back and standard.

In teach back group, the patients who were not taking any prescribed medication for the past 3 months, the highest population (34.2) obtained a score of 8 . While in the patients who were taking only 1 medication, $23.07 \%$ and in patients taking more than one prescribed medications for the past 3 months $28.57 \%$ obtained a score of 8 . In the standard group, among patients who were not taking any prescribed medication the highest percent of patients (29.1) obtained a score of 7 , in patients who were taking only 1 medication obtained a score of $5(40.2 \%)$, and in patients who were taking more than one medications obtained a score of $5(57.8 \%)$. This is illustrated in Figure 3.

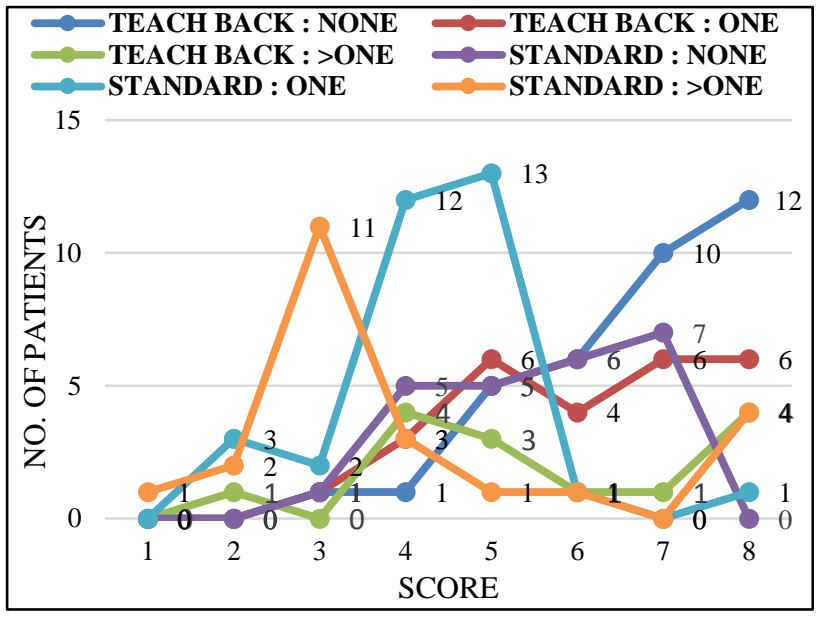

Figure 3: Drug use in past 3 months v/s score: teach back and standard. 
In the teach back group, patients who had less than high school education had a highest score of 4, patients who had less than high school education/diploma had a highest score of 5 , patients who were college graduates had a highest score of 7 , patients who had post-graduation level education had a highest score of 8 . In the standard group, patients who had less than high school education had a highest score of 4 , patients who had less than high school education/diploma had a highest score of 3 , patients who were college graduates had a highest score of 5 , patients who had post-graduation level education had a highest score of 7 (Figure 4).

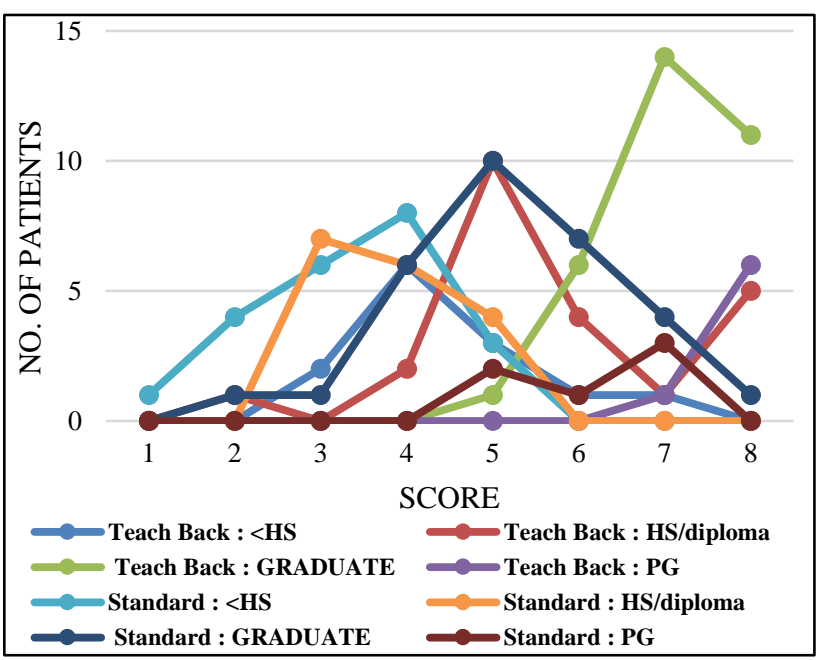

Figure 4: Education v/s score: teach back and standard.

Table 1: Test for association using Chi square Test.

\begin{tabular}{|c|c|c|c|c|}
\hline & $\begin{array}{l}\text { Teach } \\
\text { back }\end{array}$ & Standard & Total & $\begin{array}{l}\text { P Value for } \\
\text { Chi square } \\
\text { Test }\end{array}$ \\
\hline \multicolumn{5}{|l|}{ Sex } \\
\hline Male & 42 & 41 & 83 & \multirow{2}{*}{$\mathrm{P}=0.870$} \\
\hline Female & 33 & 34 & 67 & \\
\hline \multicolumn{5}{|c|}{ Age (in years) } \\
\hline $18-55$ & 54 & 49 & 103 & \multirow{2}{*}{$\mathrm{P}=0.379$} \\
\hline$>55$ & 21 & 26 & 47 & \\
\hline \multicolumn{5}{|c|}{ Rx per 3 months } \\
\hline None & 35 & 24 & 59 & \multirow{3}{*}{$\mathrm{P}=0.184$} \\
\hline One & 26 & 33 & 59 & \\
\hline$>$ One & 14 & 18 & 32 & \\
\hline \multicolumn{5}{|c|}{ Education } \\
\hline$<\mathrm{HS}^{*}$ & 13 & 24 & 37 & \multirow{4}{*}{$\mathrm{P}=0.181$} \\
\hline $\begin{array}{l}\text { HS / } \\
\text { Diploma }\end{array}$ & 23 & 16 & 39 & \\
\hline $\begin{array}{l}\text { College } \\
\text { graduate }\end{array}$ & 31 & 29 & 60 & \\
\hline $\mathrm{PG}^{* *}$ & 8 & 6 & 14 & \\
\hline
\end{tabular}

* high school, ** post graduation

None of the demographic parameters (Age, sex, drug use in past three months and education) showed any significant association with scoring and memory retention ( $\mathrm{p}$ value $>0.05$ ). This is shown in Table 1 .

In teach back method about $29.33 \%$ patients scored 8 on the other hand in standard method of counseling only $1.3 \%$ scored 8. Eight is the most frequently obtained score in teach back on contrary to standard method in which frequently obtained score was 4 (Figure 5 and Figure 6).

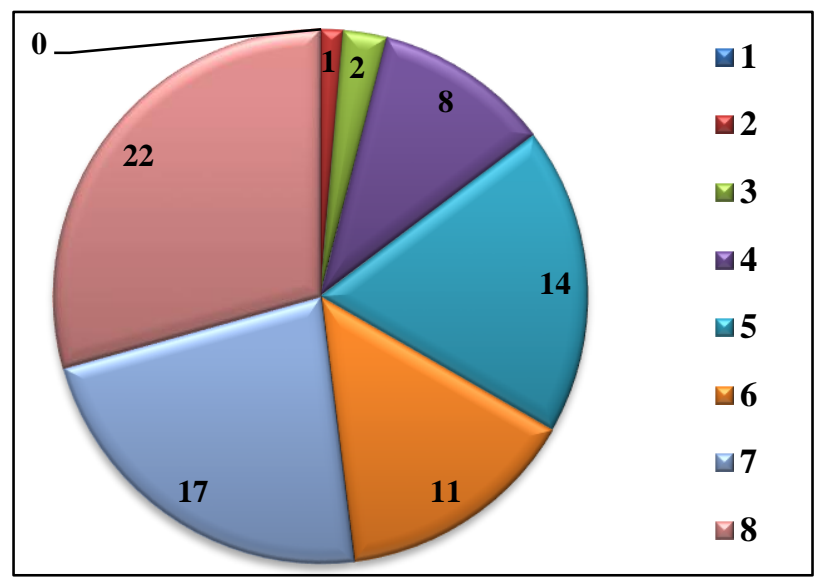

Figure 5: Teach back scoring number of patients.

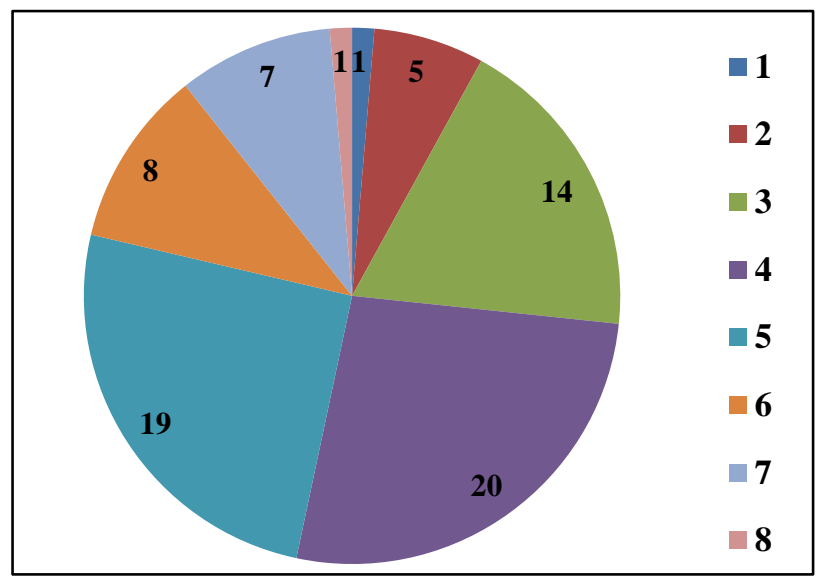

Figure 6: Standard scoring number of patients.

Table 2: Mann Whitney U Test for association between memory retention and teach back method.

\begin{tabular}{|lll|l|}
\hline & $\begin{array}{l}\text { Teach } \\
\text { back }\end{array}$ & Standard & $\begin{array}{l}\text { P Value for } \\
\text { Mann Whitney } \\
\text { U Test }\end{array}$ \\
\cline { 1 - 2 } $\mathrm{n}(\mathrm{N}=150)$ & 75 & 75 & \multirow{2}{*}{0.0001} \\
\cline { 1 - 2 } Range & $2-8$ & $1-8$ \\
\cline { 1 - 2 } Mean & 6.28 & 4.44 \\
\cline { 1 - 2 } Median & 5.5 & 4 \\
\cline { 1 - 2 } Mode & 8 & 4 & \\
\hline Mean rank & 97.91 & 53.09 & \\
\hline
\end{tabular}

Data were represented by mean rank response. Data were analyzed using Mann Whitney U test. Teach back group shows statistically significant $(\mathrm{p}$ value $=0.0001)$ increase 
in score compared to standard group. The mean value of teach back scoring is 6.28 while that of standard is 4.44 . This shows a thirty percentage increase in memory retention in the group receiving teach back method of counseling (Table 2).

\section{DISCUSSION}

Health literacy is a state where people have acquired the ability to read, understand, and use health information to make appropriate decisions concerning their health and follow correctly the instructions required for their treatment. It is stated that more than one third of the population of India lack sufficient health literacy to effectively understand and complete the needed medical treatment. Taking this fact into consideration, the health care providers have a responsibility to provide information in simple, clear and most preferably in vernacular language. Before concluding a session they should also make sure that the patient understands the information provided. The process called teach-back can be used to ensure the patient understanding. Teach-back strategies if put into practice by healthcare professionals can be a real help to all the patients especially the ones with low literacy rate.

Several studies have shown that using educational strategies increased the knowledge among the participants. A study on inpatients presented with heart failure for a time period of thirteen months reported the study sample was able to correctly answer heart failure specific teach back questions at a rate of $84.4 \% .{ }^{12}$ Authors concludes that teach-back technique is effective for both improving the outcome of COPD patient inhalation technique and can be used in different health settings. ${ }^{13}$ Our study adds to this body of evidence, signifying that implementing teach back strategies seems to perk up knowledge retention among the participants ( $\mathrm{p}$ value for Mann Whitney $U$ test was less than 0.0001).

On evaluating the relationship between age, sex, drug use and education in memory retention, a $\mathrm{p}$ value of more than 0.05 was obtained in this study which indicated the absence of statistical significance. This was similar to the observation made by previous studies. ${ }^{14}$ The time taken for patient education-which was not recorded, varied from patient to patient and this would account for the major limitation of the study since educational time interfered with memory retention. Also, the patients in the standard group themselves used collaborative teach back technique during the interactive sessions and the health-care provider was forced to use the two-way dialogue method for explaining their medications-considering the patient satisfaction, leaving the potential for bias.

The study had participants with varying degrees of literacy. It was found that all of the participants improved knowledge but that those with lower health literacy had more progress from baseline level as compared to those with higher health literacy. This finding suggests that health care providers have an opportunity to simply enhance knowledge of patients using non-expensive and on-hand educational methods, which was similar to the observation made by Negarandeh et al. ${ }^{14}$

\section{CONCLUSION}

Teach-back method is not a test of the patient's knowledge but a test of how well we have explained the concept and the patient has grasped it. In taking the extra step of asking the patients to demonstrate back, we can detect misunderstandings and thereby correct them beforehand. Incorporating this process as part of the patient-healthcare provider interaction would also increase the patients' selfcare capacity and boosts their confidence in managing their condition. Hence, teach-back method might not only improve patient understanding, but it might also positively influence patient perceptions of whether they had enough time with their health care provider.

The teach-back method is an effective method for teaching right techniques to patients coming to the pulmonary medicine department. Specific topics such as inhaler technique, nasal spray, spacers etc. when taught using teach- back method can be enhance patient understanding, which is very important for patient adherence and for improved therapeutic response.

In future the use of pictograms and the pill cards in addition to teach-back method can be studied. Implementing educational strategies to improve patient knowledge has a great impact on health outcome like medication adherence and in bringing down the administration errors of medicines.

\section{ACKNOWLEDGEMENTS}

Author would like to thank the head and staff members of the department of pulmonary medicine for providing valuable support and assistance for this study.

Funding: No funding sources

Conflict of interest: None declared

Ethical approval: The study was approved by the Institutional Ethics Committee (No: IEC/ASH/2015/PD/14)

\section{REFERENCES}

1. Hernandez LM. Standardizing Medication Labels: Confusing Patients Less, Workshop Summary. Washington, DC: The National Academic Press; 2008.

2. Ratzan SC, Parker RM, Selden CR, Zorn M, Ratzan SC, Parker RM, eds. National Library of Medicine Current Bibliographies in Medicine. Health Literacy. CBM 2000-1. Bethesda, Md.: National Institutes of Health; 2000.

3. Wolf MS, Parker RM, Ratzan SC. Literacy and public health. International Encyclopedia of Public Health: Elsevier Press; 2008:98-104. 
4. Hernandez LM. Measures of Health Literacy. Workshop summary. Washington, D.C.: The National Academic Press; 2009.

5. What Did the Doctor Say? Improving Health Literacy to Protect Patient Safety. Health Care at the Crossroads Reports series. Oakbrook Terrace, Ill.: Joint Commission; 2007. Available at: www.jointcommission.org. Accessed 28 July 2011.

6. Institute of Medicine. To Err Is Human: Building a Safer Health System. Washington, D.C.: The National Academies Press; 2000.

7. Budnitz DS, Layde PM. Outpatient drug safety: New steps in an old direction. Pharmacoepidemiol Drug Saf 2007;16(2):160-5.

8. Preventing Medication Errors. Washington, D.C.: The National Academic Press; 2006.

9. Wolf MS, Davis TC, Shrank W, Rapp DN, Bass PF, Connor UM, et al. To err is human: patient misinterpretations of prescription drug label instructions. Patient education and counseling. 2007 Aug 31;67(3):293-300.

10. Barry D. Weiss: Health literacy and patient safety: Help patient understand. Second edition. American Medical Association Foundation and American Medical Association; 2007:1-62.

11. Bourbeau J, Julien M, Maltais F, Rouleau M, Beaupré A, Bégin R, et al. Reduction of hospital utilization in patients with chronic obstructive pulmonary disease: a disease-specific self-management intervention. Archives of Internal Medicine. 2003 Mar 10;163(5):585-91.

12. White M, Garbez, R, Carroll M, Brinker E, -Esquivel J. Is Teach-Back Associated With Knowledge Retention and Hospital Readmission in Hospitalized Heart Failure Patients?. Journal of Cardiovascular Nursing. 2013;28(2):137Y146.

13. Dantic D. A critical review of the effectiveness of 'teach-back' technique in teaching COPD patients selfmanagement using respiratory inhalers. Health Edu J. 2013;73(1):41-50.

14. Negarandeh R, Mahmoodi H, Noktehdan H, Heshmat $\mathrm{R}$, Shakibazadeh E. Teach back and pictorial image educational strategies on knowledge about diabetes and medication/dietary adherence among low health literate patients with type 2 diabetes Primary Care Diabetes. 2013;7(2):111-8.

Cite this article as: Mathew MR, Mohan L, Paul M, Maideen M, Jose L, Ommanakuttan M. Evaluating effectiveness of patient counseling, teach back versus standard method. Int J Basic Clin Pharmacol 2018;7:87-92. 